\title{
Application Of Image Edge Detection In Underwater High Speed Vehicle Classification
}

\author{
Sen Wang ${ }^{\dagger}$ Qijun Liu, Jiaxing Qiu \\ Underwater Acoustics Center (Navy Submarine Academy), Qingdao, 266000, China
}

Keywords: Edge Detection, Spectrogram of Radiated Noise, Features Extraction, Classification.

\begin{abstract}
Considering the difference between high speed underwater vehicle and non-high speed underwater vehicle in the spectrogram of radiated noise, the researches on image edge detection and features extraction has been done. The SCQS(Straight-line Characteristic Quantity of Spectrogram) is definition. A method, based on image edge detection and features extraction, is put forward to get the characteristic quantity of high speed underwater vehicle and applied to researches of classification of high speed underwater vehicle. The experimental results show that the method, which can extract the features of spectrogram well, can provide a dependable characteristic to classification and early-warning of high speed underwater vehicle.
\end{abstract}

\section{Introduction}

Nowadays, many countries pay more attention to the research of high speed underwater vehicle classification and recognition. The key point of high speed underwater vehicle recognition is extract the features of high speed underwater vehicle radiated noise, distinguishing from the non-high speed underwater vehicle.

The gray change of image can be measured and located by Edge Detection. Diffuse Edge Detection can preserve the image edges while smoothing out the mixed noise[2]. Edge Linking can link the correct edge of image by autonomous edge growing and restrained growing operation without increasing the noises in the same time[3]. Though Edge Detection is extensive applied in image processing and computer vision, it is relative lack of application research on extracting the features of spectrogram of high speed underwater vehicle' radiated noise.

Classification and recognition of high speed underwater vehicle, which needs a comprehensive application of different kinds of classification methods, is a complex process. Considering the difference of spectrogram of radiated noise between high speed underwater vehicle and non-high speed underwater vehicle, the researches on image edge detection and features extraction has been done. The SCQS of vehicle radiated noise is definition. A method, based on image edge detection and features extraction, is put forward to get the SCQS of high speed underwater vehicle and applied to researches of recognition of high speed underwater vehicle.
The experimental results show that the method, which can extract the features of spectrogram well, can provide a dependable characteristic to classification and recognition of high speed underwater vehicle.

\section{Analysis of spectrogram}

\subsection{Power spectrum of radiated noise}

The spectrum of vehicle radiated noise is made by ShortTime Fourier Transform (STFT). The spectrum, which frequency on the horizontal axis and power intensity on the vertical one, is a oscillograph that shows power intensity of vehicle radiated noise changes over frequency. The STFT of discrete signal $x(n)$ of vehicle radiated noise can be defined as

$$
S_{x}(n, \omega)=\sum_{m=-\infty}^{+\infty} x(m) \cdot \gamma(n-m) \cdot e^{-j \omega m}
$$

where $\omega$ is frequency and $\gamma(\mathrm{n})$ is analysis windows function. According to the equation (1), the spectrum of high speed underwater vehicle and non-high speed underwater vehicle can be plotted.

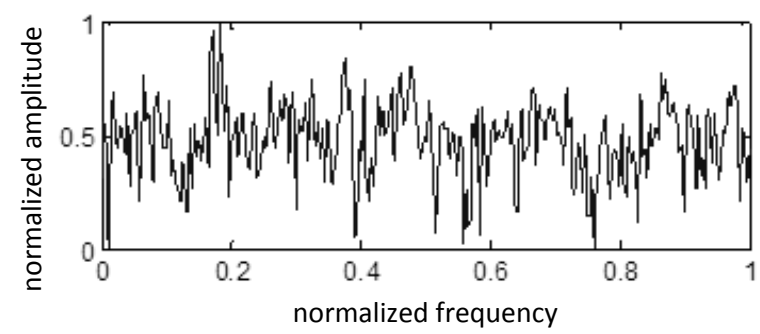

Figure 1: Spectrum of high speed underwater vehicle

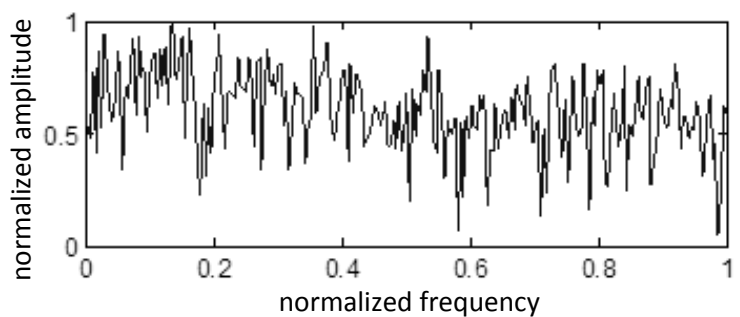

Figure 2: Spectrum of non-high speed underwater vehicle The contrast shows that the target characteristic of both spectrums is difficult to distinguish because of too much noise interference. The recognition is too hard to carry out. 


\subsection{Straight-line characteristic}

The spectrogram, which frequency on the horizontal axis and time on the vertical one, is a 2-D grey figure that shows power-spectrum of vehicle radiated noise changes over time. The matrix of spectrogram $F_{0}$, which is composed of $\mathrm{M}$ rows represent target radiated noise string of length $\mathrm{N}$, can be written :

$$
F_{0}=\left[\begin{array}{ccc}
S_{x}\left(n_{1}, \omega_{1}\right) & \cdots & S_{x}\left(n_{1}, \omega_{N}\right) \\
\vdots & \ddots & \vdots \\
S_{x}\left(n_{M}, \omega_{1}\right) & \cdots & S_{x}\left(n_{M}, \omega_{N}\right)
\end{array}\right]_{M \times N}
$$

According to the equation (2), the spectrogram of high speed underwater vehicle and non-high speed underwater vehicle can be plotted.

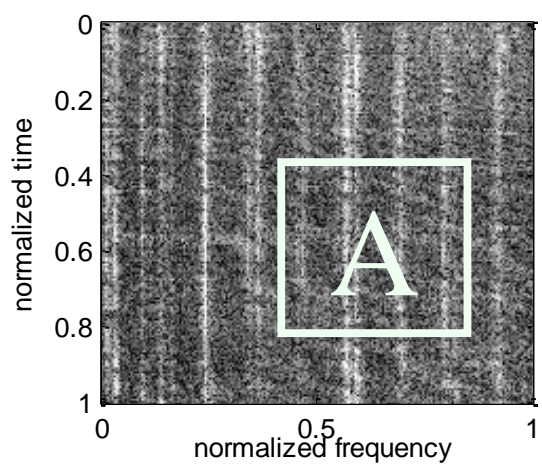

Figure 3:High speed underwater vehicle

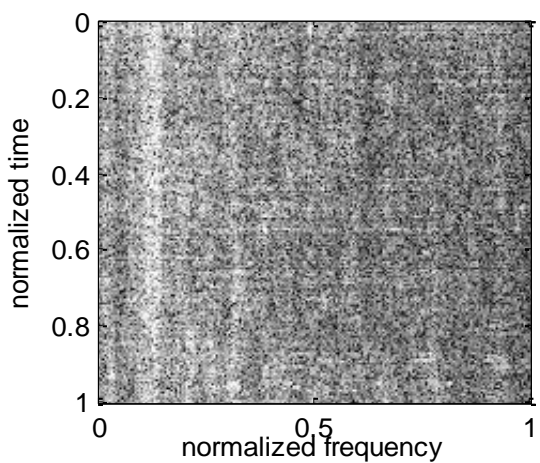

Figure 4: Non-high speed underwater vehicle

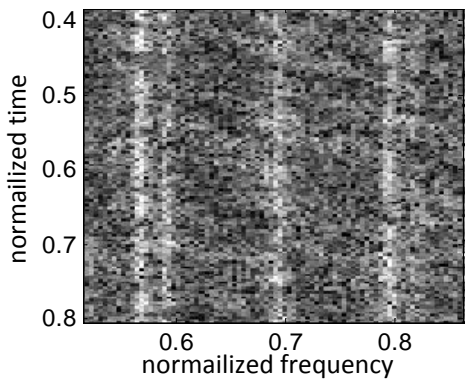

Figure 5 Partial enlarged detail of A in Fig. 3

High speed underwater vehicle, which spectrogram of radiated noise with big variation of gray level (Fig. 3, Fig.4 and Fig. 5), has a quite high rotate speed relative to non-high speed underwater vehicle.

\section{Image Edge Detection}

Extract the line features of high speed underwater vehicle spectrogram with Image Edge Detection. The flow chart is shown in Fig. 6.

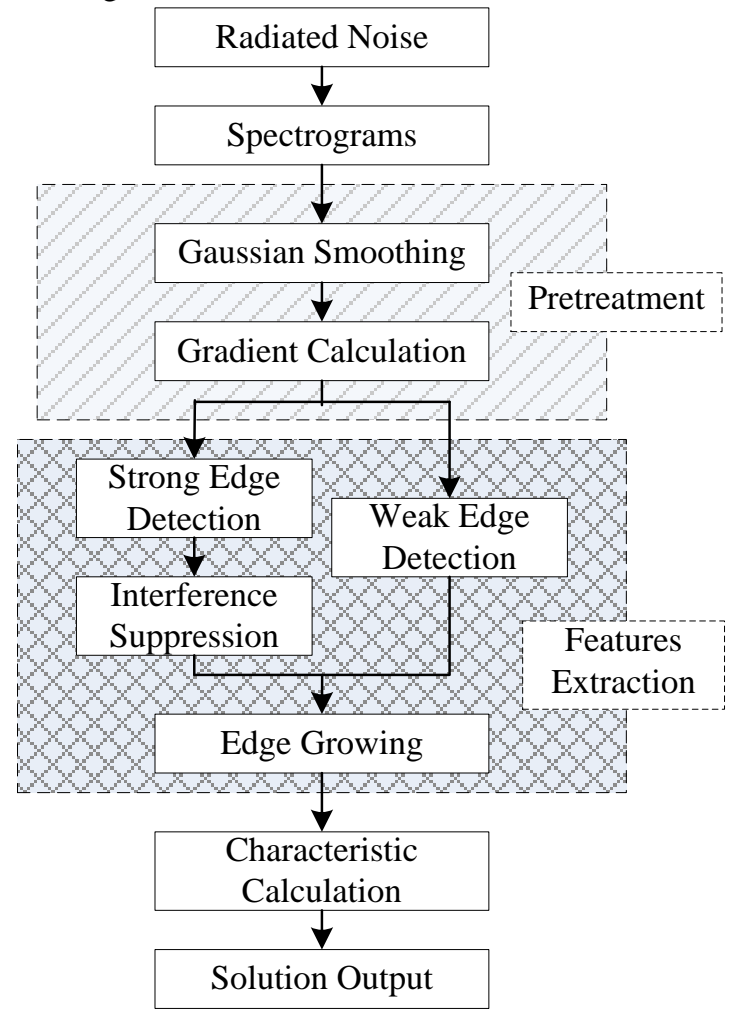

Figure 6: Flow Chart of Features Extraction

\subsection{Pretreatment}

The Gaussian smoothing matrix $F_{\text {Gauss }}$ of $F_{0}$ can be get by choosing an appropriate function.

$$
F_{\text {Gauss }}=\left[\begin{array}{ccc}
S_{\text {Gauss }}\left(n_{1}, \omega_{1}\right) & \cdots & S_{\text {Gauss }}\left(n_{1}, \omega_{N}\right) \\
\vdots & \ddots & \vdots \\
S_{\text {Gauss }}\left(n_{M}, \omega_{1}\right) & \cdots & S_{\text {Gauss }}\left(n_{M}, \omega_{N}\right)
\end{array}\right]_{M \times N}
$$

where

$$
\begin{aligned}
& S_{\text {Gauss }}\left(n, \omega_{x}\right)=S_{x}^{(0)}\left(n, \omega_{x}\right)-S_{x}^{(k+1)}\left(n, \omega_{x}\right) \\
& S_{x}^{(k+1)}\left(n, \omega_{x}\right)=\frac{1}{2 l} \sum_{i=-l}^{l} S_{x}^{(k)}\left(n, \omega_{x+i}\right)
\end{aligned}
$$

Then, matrix $\mathrm{F}$ is given

$$
F=F_{0}-F_{\text {Gauss }}=\left[\begin{array}{ccc}
S_{x}^{\prime}\left(n_{1}, \omega_{1}\right) & \cdots & S_{x}^{\prime}\left(n_{1}, \omega_{N}\right) \\
\vdots & \ddots & \vdots \\
S_{x}^{\prime}\left(n_{M}, \omega_{1}\right) & \cdots & S_{x}^{\prime}\left(n_{M}, \omega_{N}\right)
\end{array}\right]_{M \times N}
$$

The edge gradient matrix $F_{\text {grad }}$ of $F$ can be written :

$$
F_{\text {grad }}=\left[\begin{array}{ccc}
G\left(n_{1}, \omega_{1}\right) & \cdots & G\left(n_{1}, \omega_{N}\right) \\
\vdots & \ddots & \vdots \\
G\left(n_{M}, \omega_{1}\right) & \cdots & G\left(n_{M}, \omega_{N}\right)
\end{array}\right]_{M \times N}
$$

where

$$
G\left(n_{x}, \omega_{y}\right)=\left|G_{1}\left(n_{x}, \omega_{y}\right)\right|+\left|G_{2}\left(n_{x}, \omega_{y}\right)\right|
$$




$$
G_{1,2}\left(n_{x}, \omega_{y}\right)=S_{x}^{\prime}\left(n_{x}, \omega_{y}\right) * H_{1,2}
$$

Where $G\left(n_{x}, \omega_{y}\right)$ and $G_{1,2}\left(n_{x}, \omega_{y}\right)$ represent edge gradient and edge gradient component of some point in matrix $F . H_{1,2}$ are the edge detection template.

\subsection{Features Extraction}

The binaryzation of $F_{\text {grad }}$ can be done by appropriate strong and weak threshold. The strong and weak edge feature matrix $F_{S, W}$ can be given:

$$
\begin{gathered}
F_{S}=\left[\begin{array}{ccc}
G_{s}\left(n_{1}, \omega_{1}\right) & \cdots & G_{s}\left(n_{1}, \omega_{N}\right) \\
\vdots & \ddots & \vdots \\
G_{s}\left(n_{M}, \omega_{1}\right) & \cdots & G_{s}\left(n_{M}, \omega_{N}\right)
\end{array}\right]_{M \times N} \\
F_{W}=\left[\begin{array}{ccc}
G_{w}\left(n_{1}, \omega_{1}\right) & \cdots & G_{w}\left(n_{1}, \omega_{N}\right) \\
\vdots & \ddots & \vdots \\
G_{w}\left(n_{M}, \omega_{1}\right) & \cdots & G_{w}\left(n_{M}, \omega_{N}\right)
\end{array}\right]_{M \times N} \\
G_{s}\left(n_{x}, \omega_{y}\right)= \begin{cases}1 & G\left(n_{x}, \omega_{y}\right) \geq T_{s} \\
0 & G\left(n_{x}, \omega_{y}\right)<T_{s}\end{cases} \\
G_{w}\left(n_{x}, \omega_{y}\right)= \begin{cases}1 & G\left(n_{x}, \omega_{y}\right) \geq T_{w} \\
0 & G\left(n_{x}, \omega_{y}\right)<T_{w}\end{cases}
\end{gathered}
$$

where $T_{s}$ and $T_{w}\left(T_{w}<T_{s}\right)$ are strong and weak edge detection threshold.

Strong edge detection matrix $F_{S}$ only contains the strong edge feature with little fake feature (Fig. 7(a)), while weak edge detection matrix $F_{w}$ contains most edge feature with many fake feature (Fig. 7(b)).

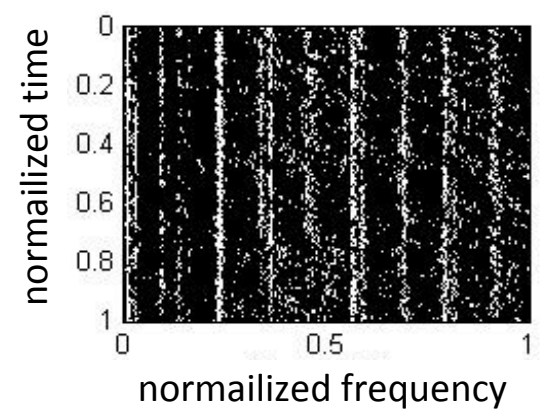

(a) Strong Edge Detection

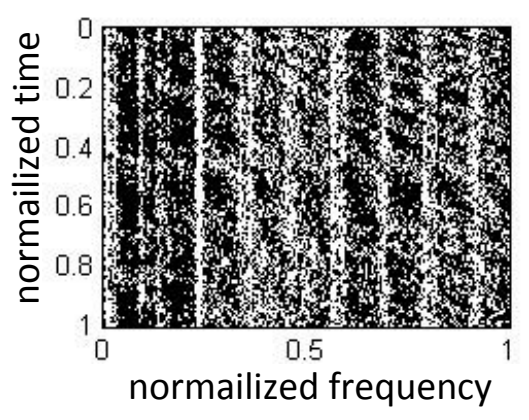

(b) Weak Edge Detection

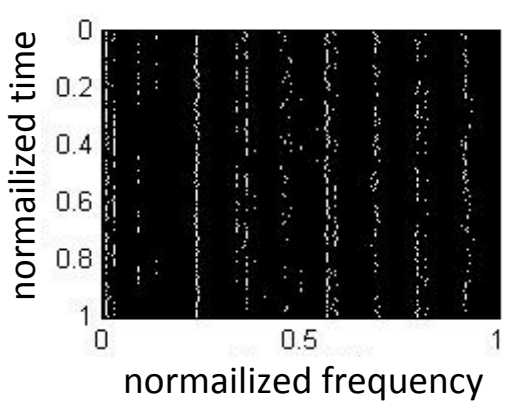

(c) Interference Suppression

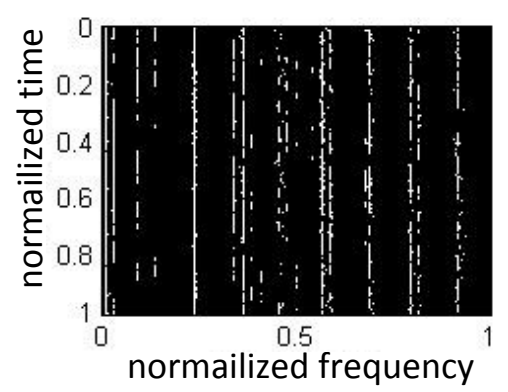

(d) Edge Growing

Figure 7: Comparison of each step of features extraction

To improve the veracity of feature extraction, the interference suppression of strong edge detection matrix $F_{S}$ must be done. Then, matrix $F_{S-D}$ can be given:

$$
F_{S-D}=F_{S} \circ D
$$

where matrix $\mathrm{D}$ is an interference suppression matrix.

The fake feature can be removed by interference suppression, while some line features are eliminated and breakages appear, as shown in Fig. 7(c). So edge growing is needed to feature extraction.

Based on the line features of spectrogram of high speed vehicle, the edge growing of the interference suppressed strong edge feature matrix $F_{S-D}$ can be done as follows:

1) Traversal search the matrix and find the Endpoints of line features.

2) Find the corresponding points of $F_{S-D}$ Endpoints in weak edge detection matrix $F_{w}$. Then, judge whether the points match the edge growing condition based on the edge growing criterion.

3) Make the edge growing of the matched points of $F_{S-D}$ with a heuristic method refering to $F_{w}$, as shown in Fig. $7(\mathrm{~d})$.

\subsection{Feature Calculation}

The SCQS is defined in order to quantize the straight-line features of high speed vehicle. The edge growed matrix $F_{\text {final }}$ of spectrogram is:

$$
F_{\text {final }}=\left[\begin{array}{ccc}
G_{f}\left(n_{1}, \omega_{1}\right) & \cdots & G_{f}\left(n_{1}, \omega_{N}\right) \\
\vdots & \ddots & \vdots \\
G_{f}\left(n_{M}, \omega_{1}\right) & \cdots & G_{f}\left(n_{M}, \omega_{N}\right)
\end{array}\right]_{M \times N}
$$

The SCQS is: 


$$
K_{S C Q S}=\alpha \cdot \frac{\sum_{i=1}^{M} \sum_{j=1}^{N} G_{f}\left(n_{i}, \omega_{j}\right)}{M \cdot N}
$$

where $\alpha$, which relates to $F_{S-D}$, is line feature coefficient.

The characteristic quantity $K_{S C Q S}$, which reflects the straightline features of spectrogram, is a key parameter. The targets can be well classified by calculating the line features difference and choosing the appropriate $\alpha$.

\section{Analysis of actual measured radiated noise}

Extract features and calculate the SCQS of actual measured radiated noise, including 57 high speed vehicles and 98 nonhigh speed vehicles, with this paper's method. The bar chart of high speed vehicles and non-high speed vehicles are shown in in Fig. 8 .

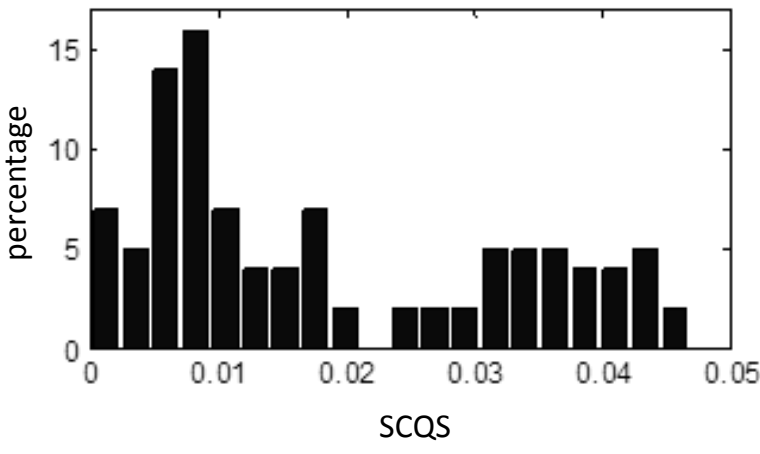

(a) High speed vehicles

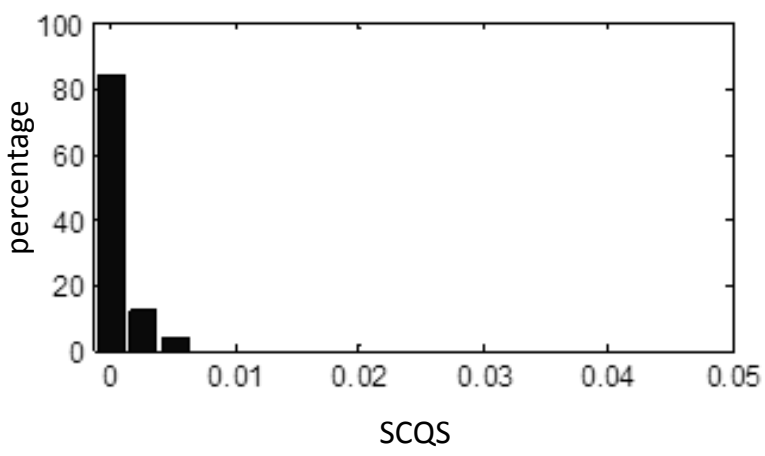

(b) Non-high speed vehicles

Figure 8: Comparison of statistics of SCQS

\begin{tabular}{|c|c|c|c|c|}
\hline & Max & Min & Mean & Variance \\
\hline $\begin{array}{c}\text { High speed } \\
\text { vehicle } \\
\begin{array}{c}\text { Non-high } \\
\text { speed vehicle }\end{array}\end{array}$ & 0.0456 & 0.0013 & 0.0183 & 0.0140 \\
\hline
\end{tabular}

Table 1: The statistics of SCQS

Following can be given from Fig.8: The SCQS of high speed vehicles, which mean is 0.0183 , is uniform distribution. But the SCQS of non-high speed vehicles, which mean is 0.0007 , is concentrated distribution about 0 . The Min SCQS of high speed vehicles is much bigger than non-high speed vehicles.
That is, high speed vehicles and non- high speed vehicles can be classified well.

\section{Conclusion}

The researches on image edge detection and features extraction has been done, considering the difference between high speed vehicles and non- high speed vehicles in the spectrogram of radiated noise. The following conclusions can be made with analysis of actual measured radiated noise:

(1) High speed vehicles, which spectrogram of radiated noise with big variation of gray level, has a quite high rotate speed relative to non-high speed vehicles. So high speed vehicles and non- high speed vehicles can be classified.

(2) The experimental results show that the method, which can extract the features of spectrogram well, can provide a dependable characteristic to recognition and early-warning of high speed vehicles.

\section{References}

[1] Dong Hongyan. "Research on Some Techniques in Edge Detection", National University of Defense Technology, (2008).

[2] Feng Ziliang, Wang Cuiqin, Shi Guanmin. "Autonomous edge growing algorithm for edge linking". Application Research of Computers, 26, pp. 3954-3956, (2009).

[3] Wang Sen, Gao Xin, Cheng Yusheng. "A Classification Method of High Speed Underwater Vehicle Based on Modulation Statistics". Acoustic Technology, 33, pp.61-64, (2014).

[4] Jiang Xiangdong. "Line spectrum distribute based high speed underwater vehicle classification method". Ship Science and Technology, 34, pp. 86-88, ( 2012).

[5] Zhou Nan. "Detection and Feature Extraction of Radiated Noise from High Speed Underwater Vehicle". Harbin Engineering University. (2011).

[6] Li Qingwu, Ma Guocui, Huo Guanying, et al. "New segmentation method of side-scan sonar image based on edge detection in NSCT domain". Chinese Journal of Scientific Instrument, 34, pp.1795-1801, (2013).

[7] Chen Qiang, Tian Jie, Liu Wei, et al. "Texture feature based target detection for SAS image". Technical Acoustics. 32, pp :273-276.(2013)

[8] Shi Meihong, Li Qing, Zhao Xueqing, et al. "A new approach for image edge detection based on conformal phase". Journal of Electronics \& Information Technology, 37, pp. 2594-2600. (2015)

[9] Wang Neng, Zhao Hongli, Ju Shenggen. "An improved adaptive Canny edge detection algorithm". Journal of Sichuan University(Natural Science Edition), 51, pp. 479-482, (2014).

[10] Nezamabadi-Pour H, Saryazdi S, Radhedi E. "Edge detection using ant algorithms". Soft Computing, 10, pp. 623-628, (2006).

[11] Wong Y P, Soh V C M, Ban K W, et al. "Improved canny edges using ant colony optimization". The Fifth International Conference on Computer Graphics, pp. 197-202, (2008). 